\title{
Secuelas Morfo-Funcionales en Mujeres Operadas de Cáncer de Mama en las Regiones de la Araucanía y del Bío-Bío, Chile
}

\author{
MorphoFunctional Consequences in Women Operated for Breast \\ Cancer of the Region of Araucanía and Bío-Bío, Chile
}

Gumiel-Urrutia, J. M. ${ }^{1,2,3}$; Burgos-Mansilla, B. ${ }^{1,4}$ \& Olave, E. ${ }^{5}$

\begin{abstract}
GUMIEL-URRUTIA, J. M.; BURGOS-MANSILLA, B. \& OLAVE, E. Secuelas morfo-funcionales en mujeres operadas de cáncer de mama en las Regiones de La Araucanía y del Bío-Bío, Chile. Int. J Morphol., 37(3):965-970, 2019.

RESUMEN: El tratamiento quirúrgico del cáncer de mama puede dejar secuelas tardías tales como escápula alada, pérdida de movilidad articular del hombro, sobrepeso, etc. Basado en lo anterior, el objetivo del presente estudio fue describir las secuelas morfofuncionales en mujeres operadas de cáncer de mama de las regiones de la Araucanía y del Bío-Bío, Chile, explorando también si el procedimiento quirúrgico conllevaría a la presencia de escápula alada. Para ello, se realizó un estudio cuantitativo, observacional y de corte transversal en treinta mujeres operadas de cáncer de mama, de edades comprendidas entre 28 y 76 años $(55,67 \pm 11,60)$. Un profesional entrenado evaluó peso, estatura, índice de masa corporal (IMC), índice de cintura cadera (ICC), rangos articulares de hombro (ROM, Range of Movement) y fuerza prensil, aplicándose además la prueba de Hoppenfeld para identificar escápula alada. Los resultados mostraron diferencias significativas en el ROM a la abducción de hombro $(\mathrm{p}<0,05)$, correlación significativa positiva de leve $(\mathrm{r}=0,370)$ a moderada $(\mathrm{r}=0,514)$ entre el ROM del lado afectado tanto para la flexión como la abducción con la fuerza prensil. Destacan, un IMC de $28,91 \pm 5,31 \mathrm{~kg} / \mathrm{m}^{2}$, un ICC de $0,86 \pm 0,06$ $\mathrm{cm}$ y la presencia de escápula alada en el 36,7 \% de las participantes. No se encontró asociación entre el abordaje quirúrgico y la presencia de escápula alada. Hubo secuelas morfo-funcionales en las mujeres en estudio, destacándose las alteraciones en el rango de movimiento del miembro superior, sobrepeso, riesgo cardiovascular y la presencia de escápula alada, sin asociarse al tipo de abordaje quirúrgico.
\end{abstract}

PALABRAS CLAVE: Cáncer de mama; Mastectomía; Secuelas; Escápula alada.

\section{INTRODUCCIÓN}

Las enfermedades neoplásicas son una problemática de salud a nivel mundial (International Agency for Research on Cancer, 2012). El cáncer de mama (CM) es el más frecuente entre las mujeres occidentales (Ferlay et al., 2015). Se estima un riesgo de por vida de desarrollar esta enfermedad de $12,15 \%$ o 1 de cada 8 mujeres (American Cancer Society, Surveillance Research, 2011). En Chile es la primera causa de muerte en mujeres. Tiene una prevalencia de 40.1 por cada 100.000 mujeres al año, siendo de 51,8 en mujeres de 35 a 64 años (Castillo et al., 2017).

Los tratamientos de esta condición se relacionan con secuelas morfo-funcionales en estas pacientes (Kozak et al., 2018). Las secuelas tempranas son inflamación local, infecciones en la zona de intervención quirúrgica entre otras. Por su parte, las secuelas tardías pueden ser escápula alada, pérdi- da de movilidad articular de hombro, sobrepeso, linfedema, disminución de la fuerza o dolor (Pinto e Silva et al., 2008; Mastrella et al., 2009; Rizzi et al., 2015; Belmonte et al., 2015).

La presencia de escápula alada está documentada en usuarias post operadas de CM (Bentzen \& Dische, 2000; Gomide et al., 2007; Mastrella et al., 2009; Adriaenssens et al., 2012; Mastrella et al., 2014; Nevola Teixeira et al., 2014; Rizzi et al., 2015; Belmonte et al.; Rizzi et al., 2016). Esta condición es la alteración de la posición escapular, caracterizándose por la aparición del margen medial en los movimientos del brazo sobre el hombro (Mastrella et al., 2009). En estos movimientos el músculo serrato anterior (SA) es el principal encargado de adherir la escápula al tórax (Latarjet \& Ruiz Liard, 2005). La lesión involuntaria del nervio torácico largo (NTL) durante la cirugía de CM ha sido men-

\footnotetext{
${ }^{1}$ Carrera de Kinesiología, Sede Temuco de la Universidad Autónoma de Chile.

${ }^{2}$ Programa de Magister en Ciencias, mención Morfología, Facultad de Medicina, Universidad de La Frontera, Temuco, Chile.

${ }^{3}$ Kinesiólogo Centro de Rehabilitación Deportiva, Kinexpert, Temuco, Chile.

${ }^{4}$ Investigador Centro de Investigación de la Araucanía (CIMA), Temuco, Chile.

${ }^{5}$ Facultad de Medicina, universidad de La Frontera, Chile.

Esta investigación fue financiada con los Fondos Internos DIUA de la Universidad Autónoma de Chile, proyecto DIUA 114-2017
} 
cionada por Rizzi et al. (2016), nervio que inerva al músculo SA. Esta lesión podría asociarse al posicionamiento prolongado del brazo en abducción pasiva, lesión directa por íntima relación con linfonodo afectado y/o por uso inadecuado del electrocauterio (Mastrella et al., 2014; Belmonte et al.). Otras funciones afectadas del músculo serrato anterior son la protracción y rotación lateral de la escápula. Esta debilidad del músculo también causaría limitaciones en la flexión y abducción del hombro, alterando el ROM (Rizzi et al., 2015). Asimismo, también existe disminución de la fuerza del resto de la extremidad (Kozak et al.).

Dentro de los abordajes quirúrgicos más usados está la linfadenectomía axilar (LA) y biopsia de linfonodo centinela (BLNC), entre otros. Durante mucho tiempo la LA fue el tratamiento de elección. En el último tiempo las cirugías han sido menos radicales conservando linfonodos axilares (BLNC) de acuerdo con su estado (Rizzi et al., 2015; Kozak et al.). Las secuelas serían menores según la literatura en el caso de la BLNC (Pinto e Silva et al.; Gomide et al.).

Otro hallazgo reportado luego de cirugía de cáncer de mama es un elevado índice de masa corporal (IMC) e índice de cintura cadera (ICC) (Vega-Malagón et al., 2014; Hernández et al., 2018). Ambas son medidas objetivas para el sobrepeso, el cual se ha asociado con riesgo de padecer cáncer. Por otro lado, linfedema post tratamiento es otra consecuencia importante, y ocurre entre el $15 \%$ al $20 \%$ de los usuarios post intervención quirúrgica y del $6 \%$ al $30 \%$ con el tratamiento conservador (Cuadrado et al., 2016).

Las consecuencias previamente descritas no están reportadas a nivel local. Es importante conocer la incidencia de estas complicaciones para adaptar los tratamientos y promover una rehabilitación funcional lo más pronta posible. Además, conocer el estado funcional y antropométrico de las mujeres post cirugía permite planificar de mejor forma su rehabilitación. Por lo tanto, el objetivo de este estudio es describir las secuelas morfo-funcionales en mujeres Chilenas, operadas de cáncer de mama de la región de la Araucanía y Del Bío-Bío. Además, explorar si el tipo intervención quirúrgica está relacionada con la presencia de escápula alada.

\section{MATERIAL Y MÉTODO}

Se realizó un estudio cuantitativo, observacional y de corte transversal, durante el año 2018. En relación a los participantes, se calculó el tamaño muestral considerando un nivel de confianza de $95 \%$, un error esperado del $10 \%$ y una incidencia esperada de $18 \%$. Se evaluó a 30 mujeres entre 28 y 76 años (MEDIA 55,67+11,60 años) operadas de CM. Fueron seleccionadas de manera no probabilística intencionada.

Las participantes provenían tanto del sistema público como privado y cumplieron con los siguientes criterios de inclusión: a) Ser mujer mayor de edad; b) Haber sido sometida a una mastectomía parcial o total; c) Pertenecer a la región de la Araucanía o del Bío- Bío. Por otro lado, se excluyeron a las usuarias que presentaron: a) Lesiones de miembro superior previo a la cirugía; b) Lesiones neurológicas que cursen con afección motora general. En relación con la data de la cirugía, la mayor parte de las participantes fueron intervenidas entre 2017 y 2018 representando a un $70 \%$ de la muestra. Respecto a la lateralidad de la cirugía, el $47 \%$ fue derecha y el $53 \%$ izquierda. En cuanto a su dominancia el $96,7 \%$ fue derecha y un $3,3 \%$ izquierda.

Las principales terapias coadyuvantes fueron radioterapia y quimioterapia con un 43 y $47 \%$ respectivamente.

Esta investigación fue aprobada por el Comité Ético Científico de la Universidad Autónoma de Chile (acta Nro. 014-17, 13 junio 2017) y se respetó lo expuesto en la Declaración de Helsinki (World Medical Association, 2013).

Mediciones. Las evaluaciones fueron realizadas por un kinesiólogo con 9 años de experiencia clínica, en el laboratorio de Análisis del movimiento humano en las dependencias de la Universidad Autónoma de Chile, sede Temuco. Estas consistieron en variables antropométricas: peso, estatura (Seca Modelo 700, Alemania, precisión 0,1 cm), para la obtención de IMC, el cual se calcula dividiendo el peso por la talla al cuadrado. Junto a lo anterior se midió perímetro de cintura y cadera (para obtener ICC). Este es el resultado de la división del perímetro de cintura por el de cadera. Junto a esto se obtuvo perímetro de brazo relajado y en contracción con cinta antropométrica (Sunny, Chile). En todas estas mediciones se respetó el protocolo ISAK (Ross et al., 2002). Además, se aplicó la Prueba de Hoppenfeld, la cual es utilizada para determinar la presencia de escápula alada (Hoppenfeld, 2002). Posteriormente se evaluaron rangos articulares (ROM) de hombro en los movimientos de flexión y abducción utilizando goniómetro (Muir et al., 2010). Por último, se realizó la medición de fuerza prensil con dinamómetro (Baseline, USA, precisión $1 \mathrm{~kg}$ ) (Kim et al., 2014).

Procedimiento. Las usuarias fueron citadas de forma individual para su evaluación. En primer lugar, se realizó una entrevista en donde se obtuvo edad, procedencia, lateralidad y data de la cirugía. A continuación, se realizó el examen 
físico. Para el peso y talla el usuario se posicionaba en bípedo, con la mirada al frente y brazos a los costados, vistiendo un mínimo de ropa. Para los perímetros de cintura y cadera la usuaria se ubicó de frente buscando ya sea el punto más angosto en el plano coronal o sagital durante una espiración normal.

En cuanto al perímetro de brazo relajado se posicionó a la usuaria de pie, midiendo la distancia media entre el margen lateral del acromion y el margen superior de la cabeza radial. En el lugar indicado se obtiene el perímetro. Usando las mismas referencias anatómicas anteriores, la usuaria ubicó su brazo en $90^{\circ}$ de abducción de hombro y $90^{\circ}$ de flexión de codo, solicitando la contracción máxima voluntaria del grupo flexor de codo, registrándose el perímetro de brazo en contracción.

Para la prueba de Hoppenfeld, las usuarias en bipedestación debían apoyar las palmas y dedos de sus manos en la pared. El evaluador solicitó que empujaran la pared, si al realizar esa acción la escápula protruía, la prueba se consideraba positiva. Para los rangos articulares de flexión y abducción de hombro se solicitó a las usuarias que se ubicaran en posición sedente. El eje del goniómetro se ubicó dos dedos por debajo del margen lateral y externo del acromion, la barra fija siguiendo el margen axilar de la escápula y la barra móvil siguiendo el eje longitudinal del antebrazo durante ambos movimientos.

Por último, en la misma posición, sosteniendo un dinamómetro con el codo en $90^{\circ}$ de flexión y hombro en posición neutra, las usuarias debían realizar una prensión con el máximo esfuerzo posible. Todos los datos fueron recolectados por un solo evaluador, además las pruebas se repitieron al menos tres veces para obtener un margen de similitud en el valor de cada variable. Estos métodos aportaron en disminuir la presencia de sesgo en este estudio.
Análisis Estadístico. Para el análisis inferencial se utilizó el software estadístico SPSS (Statistical Package for the Social Sciences) versión 23,0 ®. Para probar si los datos poseían distribución normal se aplicó la prueba Shapiro Wilks. Según normalidad se utilizó la prueba t de Student o la U the MannWhitney para identificar diferencias de medias o medianas. Para estudiar la correlación entre las variables cuantitativas se utilizó la prueba de Pearson. Para las variables categóricas, las proporciones se compararon utilizando chi-cuadrado. Para todas estas pruebas se estableció un nivel de significancia $\mathrm{p}<0,05$.

\section{RESULTADOS}

La escápula alada (signo de Hoppenfeld positivo) estuvo presente en el 36,7 \% de las participantes. El IMC de las participantes presentó una media de $28,91 \pm 5,31 \mathrm{~kg} / \mathrm{m}^{2}$ y el ICC de 0,86 $\pm 0,06 \mathrm{~cm}$. Las diferencias de ROM en la abducción, ROM en la flexión, fuerza prensil, perímetro de brazo contraído y relajado, entre lado afectado y lado control se presentan en las Figuras 1, 2 y 3 . Solamente destacan diferencias estadísticamente significativas en el ROM a la abducción ( $\mathrm{p}=0,001)$. Al dividir la muestra según presencia de signo de Hoppenfield no se encontraron diferencias en las variables mencionadas anteriormente.

No se encontró asociación entre el abordaje quirúrgico y la presencia de escápula alada. (linfadenectomía axilar, $\mathrm{p}=1,000$ y biopsia del linfonodo centinela, $\mathrm{p}=0,272$ ) A su vez tampoco encontramos correlación significativa entre signo de Hopenfeld positivo y alguna de las variables estudiadas.

Por último, se encontró correlación significativa positiva de leve $(r=0,370)$ a moderada $(r=0,514)$ entre el ROM del lado afectado tanto para la flexión como la abducción con la dinamometría del lado afectado e indemne.
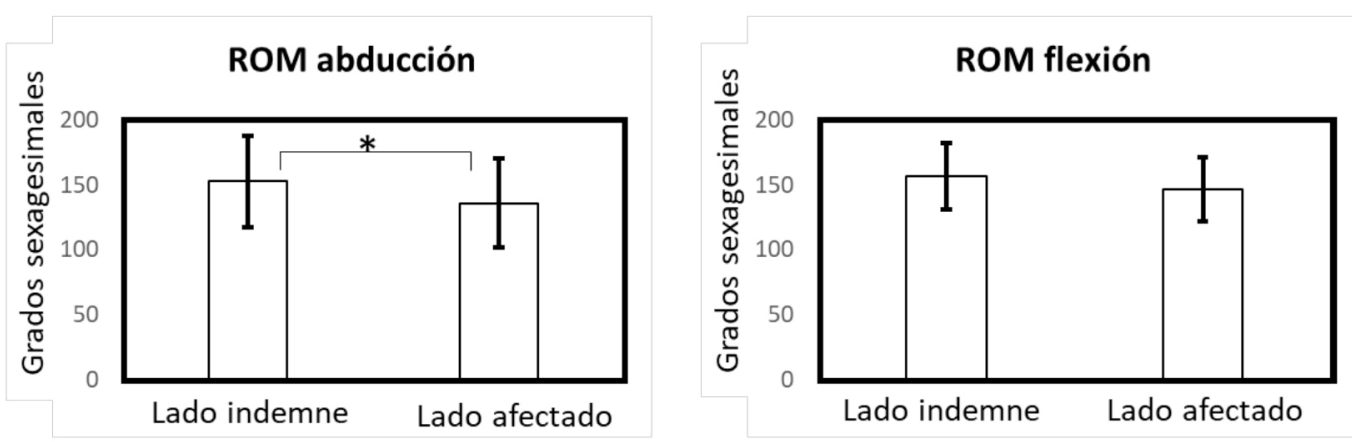

Fig. 1. Diferencias de medias y desviación estándar de rango de movimiento (ROM) entre lado afectado e indemne de los participantes $(n=30)$. Fuente: Elaboración propia con los datos obtenidos en la investigación. * Representa diferencias estadísticamente significativas $(\mathrm{p}<0,05)$. 


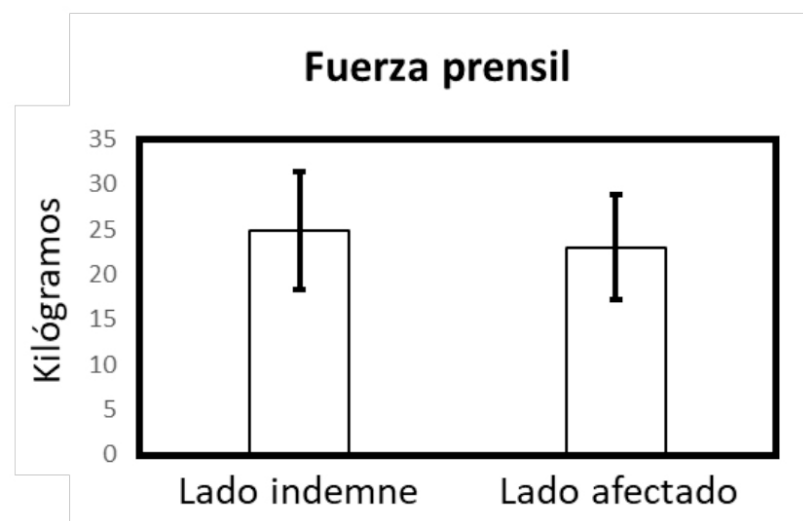

Fig. 2. Diferencias de medias y desviación estándar de fuerza prensil entre lado afectado e indemne de los participantes $(\mathrm{n}=30)$. Fuente: Elaboración propia con los datos obtenidos en la investigación. * Representa diferencias estadísticamente significativas ( $\mathrm{p}<0,05)$.

\section{DISCUSIÓN}

El objetivo de esta investigación fue describir las secuelas morfo-funcionales en mujeres operadas de cáncer de mama de la región de la Araucanía y Del Bío-Bío. En este sentido, los principales resultados demostraron una disminución significativa del ROM de abducción de hombro del lado afectado, la presencia de escápula alada, riesgo cardiovascular y sobrepeso.

Respecto a la disminución del ROM esta investigación coincide con estudios anteriores. Kozak et al. mencionaron la merma significativa del rango articular post cirugía de cáncer de mama, principalmente limitaciones en la abducción y la flexión. Adriaenssens et al. encontraron una baja 8,6 \% en la abducción post intervención quirúrgica. Rizzi et al. (2016) por otra parte, observaron alteración en movimientos de flexión, aducción, abducción, rotación interna y externa. Esta situación podría deberse a la posible fibrosis axilar y de la pared del tórax, linfedema, daño neural, vascular o musculoesquelético (Bentzen \& Dische).

La incidencia de escápula alada en nuestro estudio fue $36,7 \%$, lo que coincide con lo reportado por otros estudios (Bentzen \& Dische; Gomide et al.; Mastrella et al., 2009; Adriaenssens et al.; Mastrella et al., 2014; Nevola Teixeira et al.; Rizzi et al., 2015, Belmonte et al.; Rizzi et al., 2016). Belmonte et al. indicaron un 13,6\% de escápula alada post intervención. Así como también Mastrella et al. (2014) $28,1 \%$ y Nevola Teixeira et al. un 27,4 \%. Esto sería probablemente por retracción cutánea post intervención quirúrgica, lesión involuntaria del nervio toráxico largo, posicionamiento en abducción pasiva por mucho tiempo, lesión directa por íntima relación con linfonodo afectado y además por uso inadecuado del electrocauterio (Mastrella et al., 2009, Rizzi et al., 2015).

En nuestros resultados el $30 \%$ de las mujeres tenía sobrepeso y 43,3\% obesidad. De forma similar Ferri et al. (2010) encontraron un 44,66\% de mujeres con sobrepeso y $36,9 \%$ con obesidad. El alto IMC podría deberse a la edad de las usuarias. Se ha establecido que la edad en mujeres es determinante en el aumento del IMC (Hernández et al.). Esto se acrecienta en mujeres post menopáusicas, por la mayor producción de estrógenos en el tejido adiposo. Sumado a esto un IMC alto incrementa el riesgo de padecer carcinoma mamario en mujeres posmenopáusicas en aproximadamente un $40 \%$, además de acrecentar las posibilidades de padecer enfermedades cardiovasculares (Vega-Malagón et al.).

En cuanto al ICC, encontramos gran presencia de obesidad abdominovisceral, lo cual se asocia a un riesgo cardiovascular aumentado y a un incremento de la probabilidad de contraer enfermedades como Diabetes Mellitus
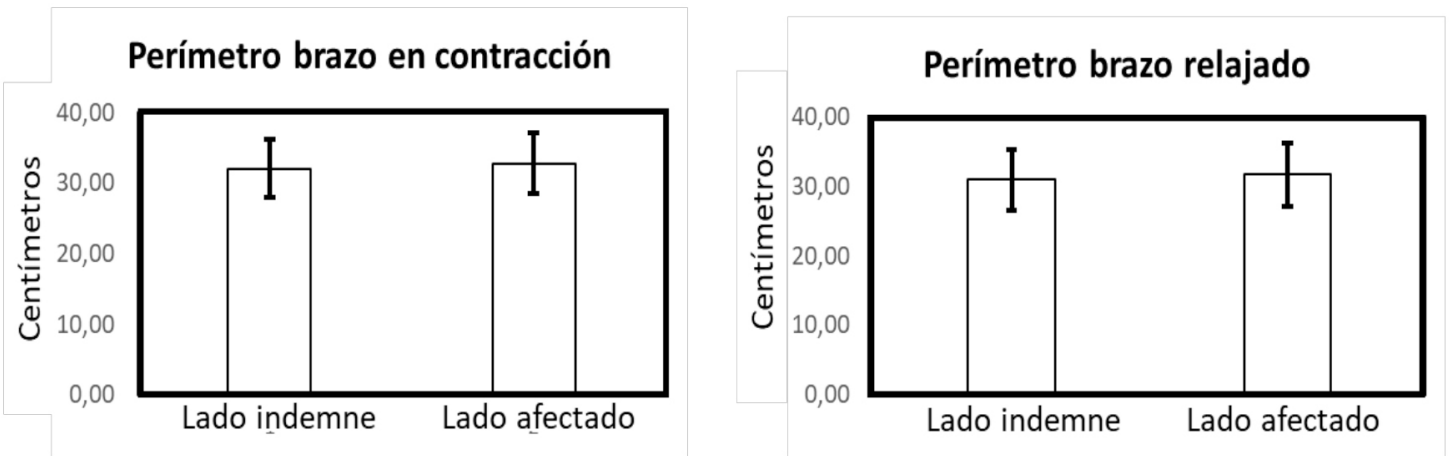

Fig. 3. Diferencias de medias y desviación estándar de perímetro de brazo entre lado afectado e indemne de los participantes $(n=30)$. Fuente: Elaboración propia con los datos obtenidos en la investigación. * Representa diferencias estadísticamente significativas $(\mathrm{p}<0,05)$. 
e Hipertensión Arterial, concordando con Vega-Malagón et al. quienes informaron de un $80 \%$ de mujeres con ICC elevado y $82 \%$ de ellas, con perímetro de cintura por sobre los $88 \mathrm{~cm}$. Lo anterior podría estar relacionado al uso de terapias de reemplazo hormonal y la edad de las usuarias. Igualmente cabe señalar que se ha estudiado separando grupos de pacientes con y sin cáncer de mama, encontrándose ICC cadera elevados en ambos grupos (Hernández et al.).

El promedio de edad de las participantes fue de $55,67+11,60$ años, en donde el 66,6 \% \% de las usuarias tenía más de 50 años. Esto coincide con lo encontrado en la literatura, ya que, por sobre los 50 años se demostró un riesgo 10 veces mayor de sufrir cáncer de mama en comparación con usuarias de 30 años (Vega-Malagón et al.). Franco-Marina et al. (2009) encontraron una relación estadísticamente significativa entre edad y cáncer de mama en mujeres sobre 44 años.

Se observó una correlación significativa positiva de leve a moderada entre el ROM del lado afectado tanto para la flexión como la abducción con la dinamometría del lado afectado e indemne, no encontramos investigaciones de similares características para comparar los análisis. Sería bueno considerar estos análisis en futuros estudios.

En este estudio, no se encontró una correlación significativa entre el abordaje quirúrgico (BLNC o AL) y la presencia de escápula alada. Similares observaciones fueron reportadas por Rizzi et al. (2016) Esto podría estar asociado al aumento de tejido adiposo subcutáneo en las usuarias (Kozak et al.) lo que dificultaría la detección de escápula alada.

Una de las principales fortalezas de esta investigación es que nos entrega información de la realidad local de las mujeres post operadas de cáncer de mama. Además, todas las evaluaciones fueron realizadas por la misma persona.

Debido al tamaño muestral de nuestros resultados es complejo generalizar los resultados. El test de Hoppenfeld es evaluador dependiente, lo que podría mejorarse en el futuro usando el test junto a electromiografía de superficie.

Nuestro estudio es importante porque describe las secuelas morfo- funcionales post cirugía en las regiones de La Araucanía y Del Bío-Bío. Creemos que esta información permite a los profesionales implicados en el tratamiento de las usuarias con esta condición, prevenir como tratar estas secuelas a tiempo.
Sería interesante poder replicar este estudio aumentando el tamaño de la muestra y el número de variables a medir, así también proponer protocolos de tratamiento para estas consecuencias a través de programas integrados de rehabilitación física.

En resumen, las mujeres operadas de cáncer de mama de la región de la Araucanía y del Bío-Bío presentaron secuelas morfo-funcionales, destacando las alteraciones en el rango de movimiento del miembro superior, sobrepeso, riesgo cardiovascular y presencia de escápula alada. Así mismo, no hubo diferencia estadística entre la presencia de escápula alada y el tipo de cirugía de cáncer de mama.

GUMIEL-URRUTIA, J. M.; BURGOS-MANSILLA, B. \& OLAVE, E. Morphofunctional consequences in women operated for breast cancer of the Regions of Araucanía and Bío-Bío, Chile. Int. J. Morphol., 37(3):965-970, 2019

SUMMARY: Surgical treatment of breast cancer can leave late sequelae such as winged scapula, loss of joint mobility of the shoulder, overweight, etc. Based on the above, the objective of the present study was to describe the morpho-functional sequelae in women operated on for breast cancer from the regions of Araucanía and Del BíoBío, Chile, also exploring whether the surgical procedure would lead to the presence of scapula winged. For this, a quantitative, observational and cross-sectional study was conducted in thirty women operated on for breast cancer, aged between 28 and 76 years $(55.67 \pm 11.60)$. A trained professional evaluated weight, height, body mass index (BMI), hip waist index (ICC), shoulder joint ranges(ROM, Range of Movement) and prehensile strength, and applied the Hoppenfeld test to identify the winged scapula. The results showed significant differences in the ROM to shoulder abduction ( $\mathrm{p}<0.05)$, positive significant correlation of mild $(r=0.370)$ to moderate $(r=0.514)$ between the ROM of the affected side for both flexion and abduction with prehensile force. Highlights, a BMI of 28.91 $\pm 5.31 \mathrm{~kg} / \mathrm{m}^{2}$, an ICC of $0.86 \pm 0.06 \mathrm{~cm}$ and the presence of winged scapula in $36.7 \%$ of the participants. No association was found between the surgical approach and the presence of the winged scapula. There were morphofunctional sequelae in the women under study, highlighting the alterations in the range of movement of the upper limb, overweight, cardiovascular risk and the presence of the winged scapula, without being associated with the type of surgical approach.

KEY WORDS: Breast cancer; Mastectomy; Sequelae; Winged scapula. 


\section{REFERENCIAS BIBLIOGRÁFICAS}

Adriaenssens, N.; De Ridder, M.; Lievens, P.; Van Parijs, H.; Vanhoeij, M.; Miedema, G.; Voordeckers, M.; Versmessen, H.; Storme, G.; Lamote, $\mathrm{J}$.; et al. Scapula alata in early breast cancer patients enrolled in a randomized clinical trial of post-surgery short-course image-guided radiotherapy. World J. Surg. Oncol., 10:86, 2012.

American Cancer Society. Surveillance Research, 2011.

Belmonte, R.; Monleon, S.; Bofill, N.; Alvarado, M. L.; Espadaler, J. \& Royo, I. Long thoracic nerve injury in breast cancer patients treated with axillary lymph node dissection. Support Care Cancer, 23(1):16975, 2015.

Bentzen, S. \& Dische, S. Morbilidad relacionada con la irradiación axilar en el tratamiento del cáncer de mama. Acta Oncol., 39(3):337-47, 2000.

Castillo, C. S. M.; Cabrera, M. E. C.; Derio, P. L.; Gaete, V. F. \& Cavada, C. G. Resultados del tratamiento del cáncer de mama, Programa Nacional de Cáncer del Adulto. Rev. Med. Chile, 145(12):1507-13, 2017.

Cuadrado, G. A.; Andrade, M. F. C.; Akamatsu, F. E. \& Jacomo, A. L. Anatomy of the lymphatic drainage of the upper limb and breast and its role in lymphedema prevention after breast cancer treatment. Int. J. Morphol., 34(3):1117-22, 2016.

Ferlay, J.; Soerjomataram, I.; Dikshit, R.; Eser, S.; Mathers, C.; Rebelo, M.; Parkin, D. M.; Forman, D. \& Bray, F. Cancer incidence and mortality worldwide: sources, methods and major patterns in GLOBOCAN 2012. Int. J. Cancer, 136(5):E359-86, 2015.

Ferri, N. N.; Ferri, A. F. A.; Ferri, A. F. A.; Brunicardi, H. R. A.; Franco, T. A. E. \& Franco, M. A. L. Cáncer de mama e índice de masa corporal: en mujeres posmenopáusicas. Rev. Venez. Oncol., 22(2):94-102, 2010.

Franco-Marina, F.; Lazcano-Ponce, E. \& López-Carrillo, L. Breast cancer mortality in Mexico: an age-period-cohort analysis. Salud Publica Mex., 51 Suppl. 2:s157-64, 2009.

Gomide, L. B.; Matheus, J. P. \& Candido dos Reis, F. J. Morbidity after breast cancer treatment and physiotherapeutic performance. Int. J. Clin. Pract., 61(6):972-82, 2007.

Hernández, R. J.; Moncada, E. O. M. \& Arnold, D. Y. Usefulness of the waist/hip index in the detection of cardiometabolic risk in overweight and obese individuals. Rev. Cuba. Endocrinol., 29(2):1-16, 2018.

Hoppenfeld, S. Exame do Ombro. In: Hoppenfeld, S. Propedêutica Ortopédica Coluna e Extremidades. Rio de Janeiro, Atheneu, 2002.

International Agency for Research on Cancer (IARC). The GLOBOCAN Project. Cancer Incidence and Mortality Worldwide. Lyon, International Agency for Research on Cancer (IARC), 2012. Disponible en: http:// globocan.iarc.fr

Kim, J. K.; Park, M. G. \& Shin, S. J. What is the minimum clinically important difference in grip strength? Clin. Orthop. Relat. Res., 472(8):2536-41, 2014.

Kozak, D.; G?owacka-Mrotek, I.; Nowikiewicz, T.; Siedlecki, Z.; Hagner, W.; Sowa, M. \& Zegarski, W. Analysis of Undesirable Sequelae of Sentinel Node Surgery in Breast Cancer Patients - a Prospective Cohort Study. Pathol. Oncol. Res., 24(4):891-7, 2018.

Latarjet, M. \& Ruíz Liard, A. Anatomía Humana. Vol. 1. $4^{\mathrm{a}}$ ed. Barcelona. Médica Panamericana, 2005.

Mastrella, A. de S.; Freitas-Junior, R.; Paulinelli, R. R. \& Soares, L. R. Incidence and risk factors for winged scapula after surgical treatment for breast cancer. J. Clin. Nurs., 23(17-18):2525-32, 2014.

Mastrella, A. S.; Freitas-Junior, R.; Paulinelli, R. R. \& Soares, L. R. Escápula alada pós-linfadenectomia no tratamento do câncer de mama. Rev. Bras. Cancerol., 55(4):397-404, 2009.

Muir, S. W.; Corea, C. L. \& Beaupre, L. Evaluating change in clinical status: reliability and measures of agreement for the assessment of glenohumeral range of motion. N. Am. J. Sports Phys. Ther., 5(3):98110, 2010.

Nevola Teixeira, L. F.; Lohsiriwat, V.; Schorr, M. C.; Luini, A.; Galimberti, V.; Rietjens, M.; Garusi, C.; Gandini, S.; Sarian, L. O.; Sandrin, F.; et $a l$. Incidence, predictive factors, and prognosis for winged scapula in breast cancer patients after axillary dissection. Support Care Cancer, 22(6):1611-7, 2014

Pinto e Silva, M. P.; Simioni, F. C.; Oliviera, R. R.; Piassarolli, V. P.; Colucci, L. V. \& Lange, L. D. Comparação das morbidades pós-operatórias em mulheres submetidas à linfadenectomia axilar e biópsia do linfonodo sentinela por câncer de mama - revisão de literatura. Rev. Bras. Cancerol., 54(2):185-92, 2008.

Rizzi, S. K.; Haddad, C. A.; Giron, P. S.; Pinheiro, T. L.; Nazário, A. C. \& Facina, G. Winged scapula incidence and upper limb morbidity after surgery for breast cancer with axillary dissection. Support Care Cancer, 24(6):2707-15, 2016.

Rizzi, S.; Haddad, C.; Girón, P.; Pinheiro, L.; Nazario, A. \& Facina, G. Incidência da escápula alada e morbidade dos membros superiores de pacientes no pós-operatório do tratamento do câncer de mama com abordagem axilar. Dissertação Mestrado. São Paulo, Escola Paulista de Medicina, Universidade Federal de São Paulo (UNIFESP), 2015.

Ross, W. D.; Carr, R. V.; Carter, J. E. L. \& Guelke, J. Anthropometry Fundamentals. An Illustrated Guide for Precision Anthropometry. Turnpike, Electronic Publications Inc., 2002.

Vega-Malagón, G.; Ávila-Morales, J.; García-Solís, P.; Camacho-Calderón, N. \& Becerril-Santos, A. La obesidad y su relación con el cáncer de mama en una población mexicana. Eur. Sci. J., 10(3):132-40, 2014.

World Medical Association. Declaración de Helsinki de la AMM - Principios Éticos para las Investigaciones Médicas en Seres Humanos. Ferney-Voltaire, World Medical Association, 2013. Disponible en: https://www.wma.net/es/policies-post/declaracion-de-helsinki-de-laamm-principios-eticos-para-las-investigaciones-medicas-en-seres-humanos/

\section{Dirección para correspondencia:}

Dr. Enrique Olave

Facultad de Medicina

Universidad de La Frontera

Av. Francisco Salazar 01145

Temuco

CHILE

Email: enrique.olave@ufrontera.cl

Recibido : 05-02-2019

Aceptado: 27-03-2019 